\title{
TRAINEES' REPRESENTATION ON STANDING COMMITTEES
}

In the article by $\mathrm{Dr} J . \mathrm{R}$. Hamilton in the Bulletin for June 1979 (p 105) it was stated that an elected representative of the Collegiate Trainees Committee would sit on each of the Standing Committees. This is correct for the majority of Standing Committees, but in the case of the Executive and Finance Committee the procedure is different. This Committee is appointed by Council, and the Bye-Laws lay down that it must include three members of Council who are Members (not Fellows) of the College, and one of these three must be an Officer of the Collegiate Trainees Committee.

Dr Helena Waters is the Member appointed under these provisions to the Executive and Finance Committee, and a reference to the fact should have been appended to the list of Committee members published in the January Bulletin (p. 3).

\section{AMENDMENT TO REGULATIONS}

Regulation V, I: HonORARY FeLLOWSHIP

Council at its recent meeting amended the regulation governing the election of Honorary Fellows as follows:

'Honorary Fellows may be elected at, and only at the Quarterly Meeting immediately preceding Annual

General Meetings, provided that they have been recom- mended by not less than six Members of the College (personal acquaintance not being requisite) and have been approved by not less than two-thirds majority of those present and voting at a meeting of the Court of Electors.'

\section{OFFICERS OF THE DIVISIONS}

\author{
Chiltern and Thames Valley Division \\ Chairman: Dr J. Steinert \\ Secretary: Dr Diana Dickens \\ Representative Fellow on Council: Dr E. Shepherd \\ Representative Member on Council: Dr Diana Dickens \\ Executive Committee: Drs B. Alapin, B. Chester, T. Crow, \\ R. Cundall H. A. Dickinson, W. B. Knapman, Professor \\ R. G. Priest, Dr V. Wilkinson \\ Co-opted: Drs A. R. M. Freeman, J. B. McWhinnie \\ Collegiate Trainees Representatives: Drs D. Costain, K. \\ Ghaffari-Tabrizi

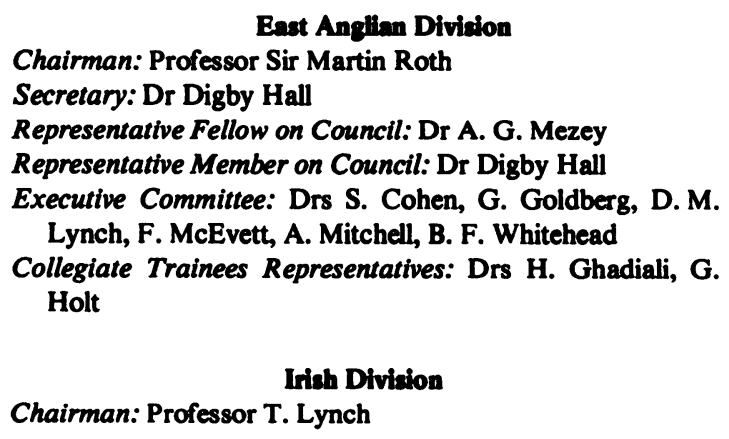

Secretary: Dr D. Shanley

Representative Fellows on Council: Drs A.S. Clenaghan, J. J. Fennelly

Representatives Members on Council: Drs D. Dunne, J. Walsh

Executive Committee: Drs P. A. Carney, M. Collins, J. Cooney, J. Corbett, R. Draper, J. Egan, J. J. Fennelly, R. Galloway, M. Hartmann, J. Ledwith, S. Martens, Professor P. J. Meehan, Drs W. B. McConnell, S. D. McGrath, P. McQuaid, W. M. Nelson, J. O'Boyle, U. O'Donnell, C. Patton, C. Smith, V. Sullivan, P. Turbridy, J. Walsh, D. Walshe, Professor M. Webb

Collegiate Trainees Representatives: Drs J. O'Riordan, J. Scott

Chairman: Dr C. P. Seager

Midland Diviaion

Secretary: Dr L. I. Liebling

Representative Fellow on Council: Dr C. P. Seager

Representative Member on Councll: Dr L. I. Liebling

Executive Committee: Dr C. Berry, Professor R. Bluglass, Drs P. Crofts, T. L. Dunn, M. A. Ezzat, D. Gill, E. B. Gordon, P. Morris, A. H. Ogden, B. E. Oliver, R. L. Palmer, K. Raychaudhuri, L. G. Scarth 